\title{
Resultados do acompanhamento dos usuários do Centro de Atenção Psicossocial - Álcool e Drogas (CAPs-AD)
}

\author{
Rivelilson Mendes de Freitas ${ }^{1}$ \\ Helen Rute Rodrigues da Silva ${ }^{2}$ \\ Diego Santos de Araújo ${ }^{3}$
}

\begin{abstract}
Neste trabalho, delinearam-se os problemas de saúde, as hipóteses diagnósticas, as substâncias psicoativas utilizadas e se há ou não aderência aos tratamentos entre os pacientes atendidos pelo Caps. O estudo, do tipo exploratório descritivo, com abordagem quantitativa, foi realizado por meio de uma entrevista com todos os pacientes $(n=31)$ em regime de hospitalidade-dia no Caps, antes das suas consultas ambulatoriais. Dos pacientes, 35,5\% tinham idade superior a 41 anos, $83,9 \%$ a cor da pele autorreferida branca e $71 \%$ eram solteiros, $74,2 \%$ tinham apenas o ensino fundamental e $48,3 \%$ tinham renda mensal de até um salário-mínimo. O principal problema de saúde identificado foi o etilismo e a hipótese diagnóstica mais comum foi o tabagismo associado ao etilismo. A maioria dos usuários administra seus medicamentos no horário certo e não pratica automedicação.
\end{abstract}

Descritores: Saúde Pública; Epidemiologia; Transtornos Mentais, Bebidas Alcoólicas; Drogas Ilícitas.

\footnotetext{
${ }^{1}$ PhD, Professor Adjunto, Universidade Federal do Piauí, Teresina, PI, Brasil.

2 Enfermeira.

${ }^{3}$ Graduando em Enfermagem, Universidade Federal do Piauí, Teresina, PI, Brasil.
}

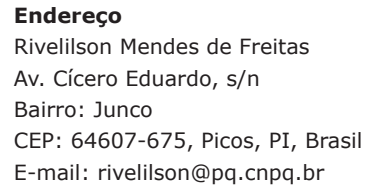




\section{Results of monitoring of users treated in the Psychosocial Care Center - Alcohol and Drugs (CAPS-AD)}

This study has drawn a line on health problems, diagnostic hypotheses, psychoactive substances used and if there is or not adherence to treatment among patients treated by the CAPS. The exploratory and descriptive research with a quantitative approach was conducted through an interview with all patients $(n=31)$ under hospitality day at CAPS before their outpatient consultations. From all of them, 35.5\% were older than 41 years, $83.9 \%$ had skin color self-reported as white and $71 \%$ were single, $74.2 \%$ had completed only primary education and $48.3 \%$ had a monthly income of up to a basic wage. The main health problem identified was alcoholism and the most common diagnostic hypothesis was smoking associated with alcoholism. Most users take their medication at the right time and do not practice self-medication.

Descriptors: Salud Pública; Epidemiología; Trastornos Mentales; Bebidas Alcohólicas; Drogas Ilícitas.

\section{Resultados del acompañamiento de los usuarios del Centro de Atención Psicosocial - Alcohol y Drogas (CAPS - AD)}

El trabajo delineó los problemas de salud, las hipótesis diagnósticas, las substancias psicoactivas utilizadas y se hay o no adherencia a los tratamientos entre los pacientes atendidos por el CAPS. El estudio del tipo exploratorio descriptivo en abordaje cuantitativo fue realizado, por medio de una entrevista con todos los pacientes $(n=31)$ en régimen de hospitalidad día en el CAPS antes de sus consultas de ambulatorio. 35,5\% de los pacientes tenían edad superior a 41 años, $83,9 \%$ el color de la piel auto-referida blanca y $71 \%$ eran solteros. $74,2 \%$ tenían apenas la enseñanza fundamental y 48,3\% tenían renta mensual de hasta un salario. El principal problema de salud identificado fue el etilismo y la hipótesis diagnóstica más común fue el tabaquismo asociado al etilismo. La mayoría de los usuarios administra sus medicamentos en la hora cierta y no practica automedicación.

Descriptores: Public Health; Epidemiology; Mental Disorders; Alcoholic Beverages; Street Drugs.

\section{Introdução}

Desde os anos 80, por meio da Reforma Psiquiátrica, o Brasil vem construindo uma nova forma de cuidar dos pacientes portadores de transtornos mentais, na busca para reinventar a clínica com a construção de novas subjetividades, como possibilidade de responsabilizarse pelo sofrimento de sujeitos, por meio de um novo paradigma centrado em um cuidar humano, solidário, afetivo e na tentativa de reconstruir a cidadania desses pacientes. Essa Reforma visou a substituição dos hospitais pisiquiátricos "manicômios" por novas modalidades de atendimento, como os hospitais-dia, os Centros de Atenção Piscossocial (Caps), Núcleos de Assistência Psicossocial e outros $^{(1-2)}$.

Os princípios do Caps constituem-se em um serviço comunitário que tem como papel cuidar de pessoas que sofrem transtornos mentais, em especial os transtornos 
crônicos. Devem ser obedecidos alguns princípios básicos, dentre os quais a responsabilização pelo acolhimento da demanda desses pacientes, garantindo a presença de profissional responsável, durante todo o período de funcionamento e criar ambiência terapêutica acolhedora no serviço que possa incluir pacientes nas atividades da unidade. Além disso, as ações do Caps devem ser dirigidas aos familiares e comprometendo-se com a construção dos projetos de inserção social. Deve, ainda, trabalhar com a ideia de gerenciamento de casos e personalizar o projeto de cada paciente ${ }^{(3)}$.

Estudos epidemiológicos e sobre os tratamentos farmacológicos, realizados anteriormente, descrevem as principais características sociodemográficas e, ainda, os problemas de saúde e as consequências de um tratamento farmacológico realizado de forma inadequada pelos usuários de um Centro de Atenção Psicossocial, localizado no Estado do Ceará ${ }^{(3)}$. Vários outros trabalhos realizados com usuários do Caps contribuem, de forma inequívoca, para demonstrar a necessidade de orientações sobre as patologias e os tratamentos farmacológicos para usuários, familiares e profissionais de saúde envolvidos nas atividades assistenciais do $\mathrm{Caps}^{(4-5)}$.

Os transtornos mentais, atualmente, gerados pelo uso de substâncias psicoativas representam graves problemas de saúde pública, que são frequentemente subdiagnosticados e indevidamente tratados ${ }^{(6)}$. Os estudos que enfocam esses transtornos visam identificar as inúmeras variáveis demográficas, fisiológicas, culturais e psicológicas intervenientes, que contribuem para o uso e dependência de substâncias ${ }^{(6-7)}$. Muitas vezes os dependentes químicos fazem uso de várias drogas, o que torna o tratamento mais difícil e piora o seu estado de saúde mental, podendo gerar várias outras consequências, como delírios, alucinações, além de outras psicoses ${ }^{(8-9)}$.

Diante dessa contextualização, o presente estudo pretendeu apresentar os principais dados sobre o perfil epidemiológico, os principais problemas de saúde, as hipóteses diagnósticas, as principais substâncias psicoativas utilizadas e se há ou não aderência aos tratamentos entre os pacientes portadores de transtornos psicossociais atendidos pelo Centro de Atenção Psicossocial -Álcool e Drogas (Caps-AD) no município de Picos, Piauí.

\section{Material e métodos}

\section{Desenho do estudo}

Os resultados foram obtidos por meio de um estudo do tipo exploratório descritivo, com abordagem quantitativa $^{(10)}$, por meio de uma entrevista realizada diretamente com o paciente, durante, aproximadamente, 20 minutos antes das suas consultas ambulatoriais. Durante as entrevistas com todos os pacientes $(n=31)$ em regime de hospitalidade-dia, no Caps-AD do município de Picos, Piauí, foi preenchido um questionário com perguntas claras e objetivas. Foram incluídos no estudo os pacientes que realizaram uma consulta ao mês e aqueles que se encontravam em regime de hospitalidade-dia no serviço de saúde do Caps-AD. As entrevistas foram realizadas durante o período de abril a novembro de 2009. Para evitar qualquer viés metodológico, não se entrevistaram os pacientes em atendimento de urgência.

\section{Local do estudo}

O Caps é um serviço de saúde aberto e comunitário do Sistema Único de Saúde (SUS). É um centro de referência e tratamento para pessoas que sofrem de transtornos mentais, cuja severidade e/ou persistência exigem cuidado intensivo, comunitário e personalizado ${ }^{(2)}$. O Caps foi instituído juntamente com os Núcleos de Assistência Psicossocial, através da Portaria SNAS n²24(3). É uma unidade de saúde local que conta com atendimento multiprofissional a uma população definida e que oferece atendimento de cuidados intermediários entre o regime ambulatorial e a internação em hospitalidade-dia ${ }^{(11-12)}$.

O Caps tem como princípio constituir um serviço comunitário que tem como papel principal cuidar de pessoas que sofrem transtornos mentais, em especial os transtornos crônicos. Além disso, as ações do Caps devem ser dirigidas aos familiares e se comprometer com a construção dos projetos de inserção social. Além de trabalhar com a ideia de gerenciamento de casos, personalizando o projeto de cada paciente. Entre os transtornos psicossociais mais prevalentes atendidos pelo Caps, segundo a Opas/OMS (2001), estão os transtornos depressivos, os transtornos associados ao uso de substâncias químicas lícitas e ilícitas, a esquizofrenia, a epilepsia, o retardo mental e os transtornos da infância e da adolescência.

\section{Coleta dos dados}

A coleta dos dados dos pacientes foi realizada pelos acadêmicos do Curso de Enfermagem da Universidade Federal do Piauí, treinados sob supervisão, sendo realizada por meio de entrevista direta com o paciente durante aproximadamente 20 minutos, antes das suas consultas. Durante a entrevista, foi preenchido um questionário, elaborado segundo a metodologia descrita por Cipolle e colaboradores ${ }^{(10)}$, com perguntas claras e objetivas, no intuito de registrar as seguintes informações: idade, cor da pele autorreferida, estado civil, escolaridade, renda familiar, problemas de saúde, hipóteses diagnósticas, as principais substâncias psicoativas utilizadas e se há ou não aderência aos tratamentos entre os pacientes portadores de transtornos psicossociais atendidos pelo Caps-AD. Os dados sobre as patologias prévias e as enfermidades associadas ao vício e/ou dependência química ou física, observadas durante as consultas, foram analisadas e registradas diretamente dos prontuários médicos. Foram considerados como problemas de saúde autorreferidos pelos pacientes durante as entrevistas os fatores associados de maior frequência durante os tratamentos, ou seja, etilismo e/ou tabagismo isolado ou associado a outros fatores.

O protocolo de pesquisa cumpriu os princípios éticos contidos na Declaração de Helsinki e as normas da Resolução 196/96, do Conselho Nacional de Saúde, tendo sido aprovado 
pelo Comitê de Ética em Pesquisa da Universidade Federal do Piauí (CAAE: 0093.0.045.000-09).

\section{Resultados}

O serviço de saúde estudado é referente ao Sistema Único de Saúde (SUS) do Estado do Piauí, mantido pela Prefeitura Municipal de Picos. Assim, esses resultados têm limitações, mas são representativos quanto aos pacientes portadores de transtornos psicossocias, atendidos nos serviços do SUS do Piauí. Dos 31 pacientes acompanhados no estudo, a maioria é do sexo masculino $(77,4 \%)$, $35,5 \%$ tinha idade superior a 41 anos (Figura 1), 83,9\% autorreferiu a cor da pele como branca e 16,1\%, parda. Quanto à situação conjugal, 71\% deles eram solteiros, $12,9 \%$ divorciados, $9,7 \%$ casados e $6,4 \%$ viúvos, sendo que $71 \%$ afirmaram ter cursado o ensino fundamental completo, $16,1 \%$ deles disseram ter cursado o ensino médio completo e $9,7 \%$ são analfabetos, $48 \%$ tinha renda familiar de até um salário-mínimo (Figura 2).

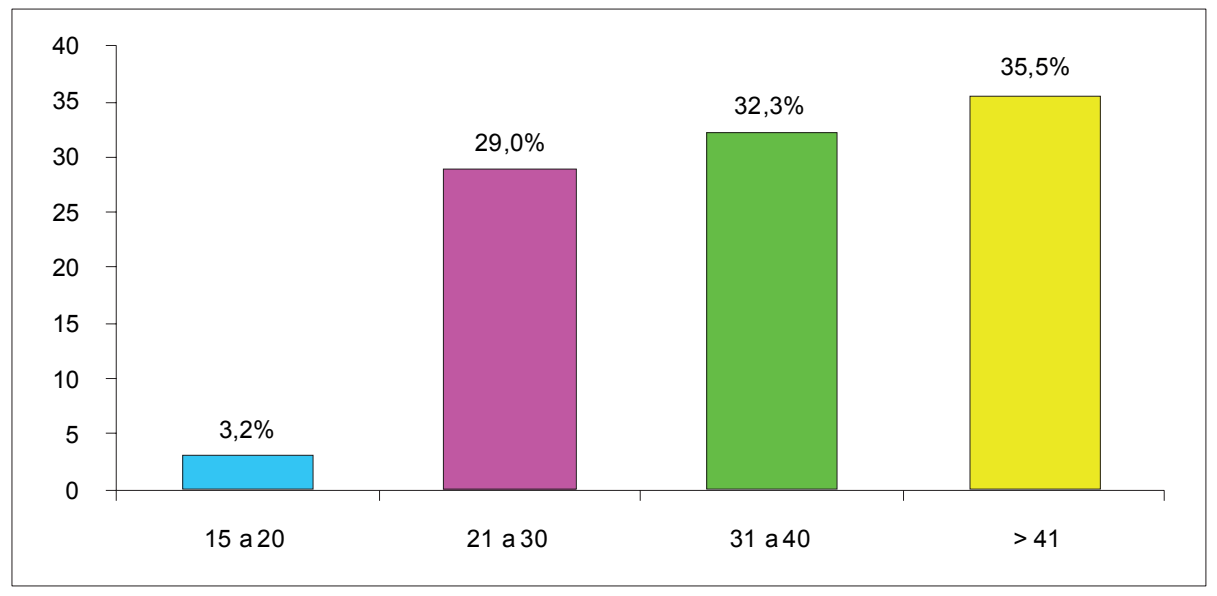

Figura 1 - Distribuição dos usuários do Caps-AD, segundo a faixa etária em anos

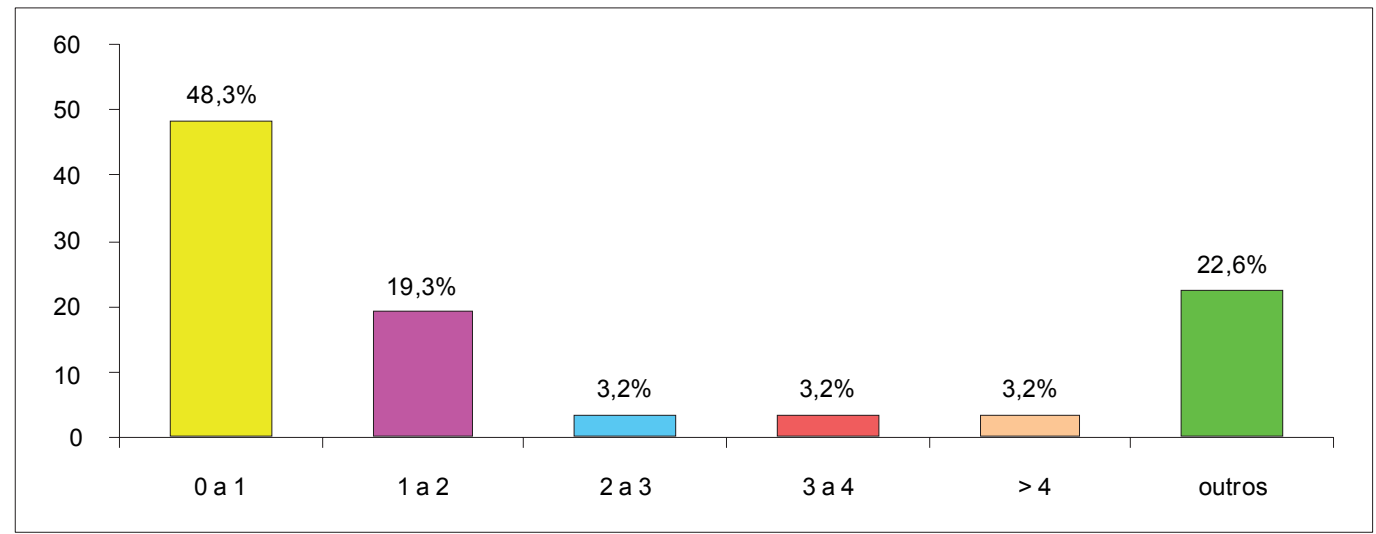

Figura 2 - Distribuição dos usuários do Caps-AD, segundo a renda familiar em salários-mínimos

Quando questionados sobre o número de filhos, $45,2 \%$ deles autorreferiram não ter filhos, $38,6 \%$ afirmaram ter de um a dois filhos e $16,1 \%$ disseram que tinham mais de dois filhos. Com relação ao tipo de moradia, 61,3\% deles possuíam moradia própria, 29\% residiam em casa alugada e 9,7\% não souberam informar o tipo de moradia.
Esses resultados tornam evidentes que, em função da baixa escolaridade, os pacientes ou exercem atividade sem remuneração ou desempenham trabalho na agricultura familiar.

O principal problema relacionado à saúde identificado foi o tabagismo, associado ao etilismo (71\%) (Figura 3). 


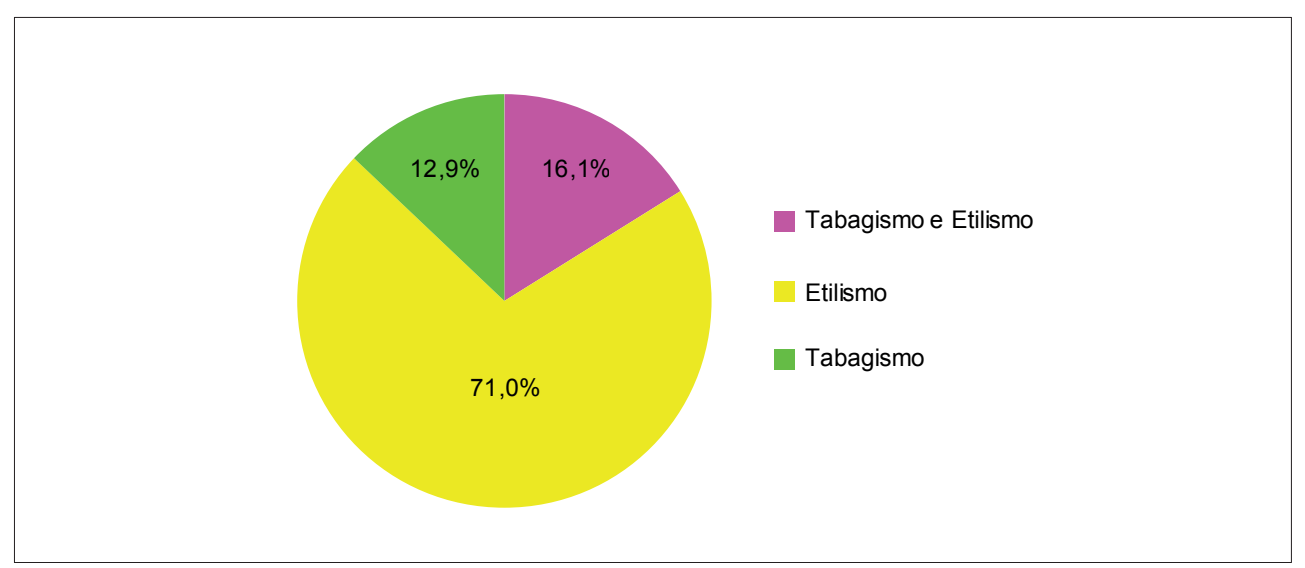

Figura 3 - Distribuição dos usuários do Caps-AD, quanto aos principais problemas relacionados à saúde

Dentre as hipóteses diagnósticas encontradas destacam-se o alcoolismo, a dependência química e o tabagismo, sendo a principal o etilismo associado ao tabagismo (45,2\%) (Figura 4). Por sua vez, a maior frequência de dependência química foi associada ao uso da maconha e crack (55,5\%) (Figura 5).

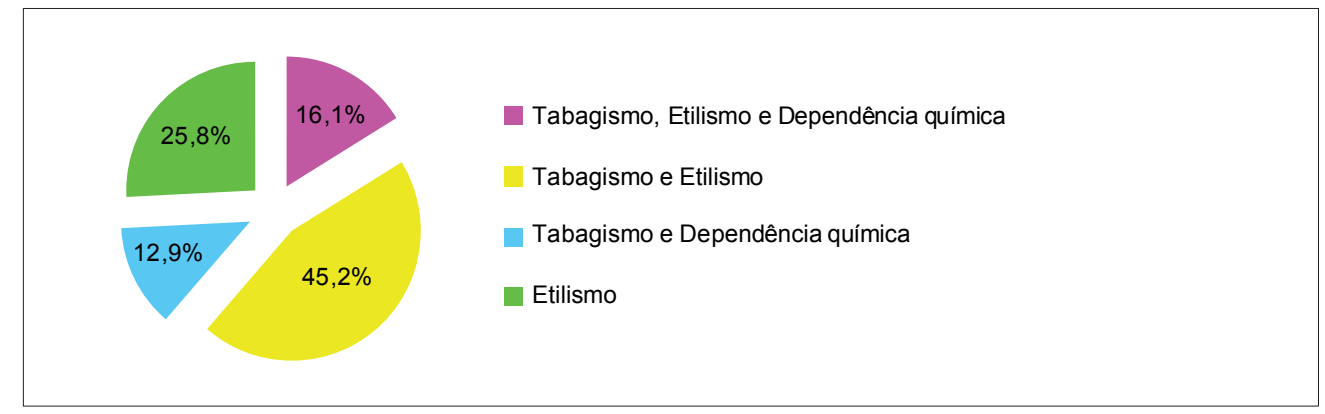

Figura 4 - Distribuição dos usuários do Caps-AD quanto às principais hipóteses diagnósticas

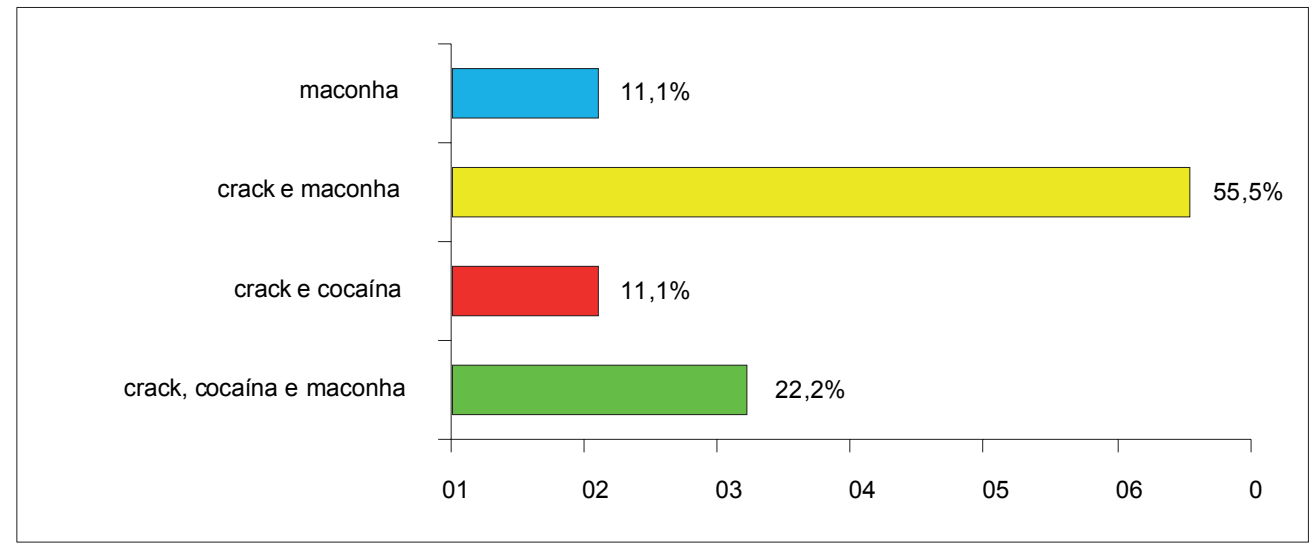

Figura 5 - Distribuição dos usuários do Caps-AD, quanto os principais tipos de dependência química 
Com relação à aderência ao tratamento farmacológico prescrito, foi verificado que a maioria dos usuários administra os seus medicamentos no horário certo (96,8\%), entretanto, 64,5\% fumam e 22,6\% bebem durante o tratamento. Por sua vez, também foi detectado que $93,6 \%$ não faziam automedicação e preferiam fazer uso de medicamentos prescritos pelo psiquiatra $(74,2 \%)$.

Neste estudo, também foi determinado que apenas pequeno número de pacientes esquece de administrar o medicamento na hora certa (12,9\%). E quando esse fato ocorre, a maior parte deles deixa para tomar a dose seguinte $(70,9 \%)$. Em geral, eles preferiam tomar a medicação com água (83,9\%), preferencialmente após as refeições $(54,8 \%)$. Outro dado importante detectado no estudo foi a baixa frequência de suspensão do tratamento após a melhora do quadro patológico $(6,4 \%)$, uma vez que, mesmo com a regressão dos sinais e sintomas dos transtornos mentais, 83,9\% continuavam fazendo uso da medicação prescrita.

\section{Discussão}

O presente estudo possibilitou identificar o perfil epidemiológico, os principais problemas de saúde, as hipóteses diagnósticas, as principais substâncias psicoativas utilizadas e se há ou não aderência aos tratamentos entre os pacientes portadores de transtornos psicossociais atendidos pelo Caps-AD, no município de Picos, Piauí.

A faixa etária superior a 40 anos de idade foi a que apresentou maior prevalência entre os usuários acompanhados no Caps, sendo essa faixa etária identificada em estudos anteriores como fator de risco para o desenvolvimento de comorbidades ${ }^{(13-14)}$. Também foi observada maior prevalência dos atendimentos com pacientes do sexo masculino, provavelmente devido a esse serviço de saúde atender principalmente os transtornos relacionados ao alcoolismo e ao vício pelas drogas que são mais frequentes entre os homens ${ }^{(15)}$.

Os transtornos mentais têm início na adolescência, na fase produtiva da vida, de inserção no mercado de trabalho e dos conflitos emocionais ${ }^{(16)}$. O "adoecer psíquico" ainda não é bem compreendido pela sociedade como uma doença de causa já bem conhecida, assim, tem a sua definição baseada não apenas em fatores biológicos, mas pela determinação cultural e de valores, o que resulta na exclusão social e isolamento desses doentes que não se enquadram dentro dos padrões habituais, dificultando, dessa forma, o seu acesso à educação e ao estabelecimento de união conjugal. Esses dados corroboram dados encontrados neste estudo, uma vez que a maioria dos pacientes não possui filhos e nem apresenta situação conjugal estável.

A maioria dos usuários possui apenas o ensino fundamental que, na maioria das vezes, é incompleto, e muitos deles não sabem ler e nem escrever corretamente, o que justifica o elevado consumo do álcool e do tabaco, uma vez que a ausência do conhecimento dos riscos e das consequências da utilização das drogas lícitas e ilícitas pode favorecer o uso dessas substâncias, bem como o bem-estar físico e psíquico que essas produzem.

No estudo, pode-se perceber que a maioria deles desenvolve atividades agrícolas, apresenta baixa escolaridade e renda familiar de aproximadamente um salário-mínimo. No entanto, o tipo de moradia mais frequente autorreferido pelos pacientes foi a casa própria, embora tenha sido relatado que os mesmos residem com seus familiares, já que não possuem condições biopsicossociais para manter um lar.

Os principais problemas, relacionados à saúde dos pacientes, determinados foram tabagismo e/ou etilismo. Por sua vez, as hipóteses diagnósticas de maior prevalência entre os pacientes são o alcoolismo, o alcoolismo associado ao vício pelas drogas, o vício pelas drogas e a dependência química. Quando questionados sobre a dependência química, foi identificado alto consumo concomitante do crack e da maconha. Entre os dependentes químicos foi verificado que pequeno número deles não faz uso do crack, revelando aumento do uso dessa substância na nossa sociedade, possivelmente devido ao seu baixo custo e ao alto potencial de causar dependência ${ }^{(17)}$.

Embora os dados, aqui, revelem situação preocupante em relação ao uso de drogas, no município de Picos, também mostram que há a procura pelos tratamentos de saúde pelos usuários e familiares, e que os pacientes, na maioria das vezes, procuram aderir ao tratamento, uma vez que apenas 3,2 e 6,4\% desses usuários autorreferiram que não administravam os medicamentos no horário certo e que realizavam a automedicação, respectivamente. Outro dado importante verificado neste estudo mostra que há preocupação com as possíveis interações entre alimentos/nutrientes com os medicamentos, uma vez que os tratamentos são administrados uma ou duas horas após as refeições e com bastante água. Embora um pequeno número de pacientes relatasse que não se esquecia de tomar o medicamento e nem fazia uso de bebidas alcoólicas, é importante ressaltar que, mesmo durante o tratamento farmacológico, número significativo de usuários fuma, mostrando que é necessária a implantação de oficinas terapêuticas e grupos de orientação farmacológica sobre os riscos e as consequências do uso de drogas lícitas e ilícitas, durante os tratamentos e para os transtornos mentais ${ }^{(18)}$.

Identificou-se, também, que os pacientes continuam o tratamento mesmo quando há melhora significativa dos sinais e sintomas dos transtornos mentais e que só fazem uso dos medicamentos prescritos pelo médico psiquiatra. Sendo assim, o acompanhamento do tratamento correto com psicofármacos visa reduzir os custos com a assistência médica e garantir maior segurança aos usuários do Caps. Pode-se definir esse acompanhamento como sendo a provisão responsável pelo tratamento farmacológico, com o propósito de alcançar resultados que melhorem a qualidade de vida do paciente ${ }^{(19-20)}$.

Esses acompanhamentos estão comprometidos com a redução da morbidade e mortalidade relacionada aos medicamentos $^{(21)}$. Na ausência desses, o processo de cura e/ou manutenção da saúde pode ser comprometida, inclusive podendo levar ao agravamento do quadro 
clínico $^{(22-23)}$, gerando, assim, transtornos para o paciente e prejuízos para os sistemas de saúde, com aumento do custo do tratamento e do número de dias de internação dos pacientes.

Dessa forma, este projeto teve como justificativa principal delinear informações necessárias para a implantação de oficinas terapêuticas e grupos de orientação farmacológica, revendo formas de abordagens e tratamentos das doenças mentais no Caps-AD do município de Picos, uma vez que a literatura enfatiza que a doença, quando diagnosticada e tratada, evita prejuízos sociais e pessoais ao paciente.

\section{Considerações finais}

É importante que os profissionais de saúde, assim como a comunidade, possam reconhecer nos pacientes com transtornos mentais seres integrais, dignos, com direito à liberdade, à integralidade física e moral, à reabilitação para o trabalho e à qualidade de vida. Dentro desse contexto, o Caps apresenta papel relevante, uma vez que é a porta de entrada do paciente ao sistema de saúde, como também apresenta a responsabilidade de propor novas estratégias de atendimento no sentido de aumentar a rede de proteção e atenção aos laços familiares.

Este estudo, com abordagem quantitativa, foi resultado da participação direta no atendimento aos usuários do Caps. Considerando tratar-se de instituição de referência para o acompanhamento dos pacientes usuários de álcool e drogas, houve o interesse em traçar o perfil da clientela assistida, acreditando-se que se poderia, então, reconhecer suas características e identificar fatores de risco que podem ser evitados ou tratados. O perfil dos usuários atendidos no Caps é de homens adultos, com baixa escolaridade, solteiros e que exercem atividade com baixa remuneração. A maioria adere ao tratamento farmacológico, não pratica automedicação e nem faz uso de bebidas alcoólicas durante o tratamento. Nesse Caps, acha-se oportuno caracterizar a população e, a partir dos resultados, ampliar suas ações para a realização de um trabalho de educação dos usuários, familiares e profissionais de saúde sobre os riscos e consequências da utilização de drogas lícitas e ilícitas.

\section{Referências}

1. Maia LC, Durante AMG, Ramos LR. Prevalência de transtornos mentais em área urbana no norte de Minas Gerais, Brasil. Rev Saúde Pública 2004;38(5):650-6.

2. Pelisoli CL, Moreira AK. Caracterização epidemiológica dos usuários do Centro de Atenção Psicossocial Casa Aberta. Rev. psiquiatr. Rio Gd. Sul 2005;27(3):270-7.

3. Oliveira CPA, Freitas RM. Instrumento Projetivo para Implantação da Atenção Farmacêutica aos Portadores de Transtornos Psicossociais: Atendidos pelo Centro de Atenção Psicossocial. SMAD, Rev. Eletrônica Saúde Mental Álcool Drog. 2008;4(2):1-15.

4. Borges KD, Iodes AF, Freitas RM. Estudo do uso racional de medicamentos por usuários do Centro de Atenção Psicossocial. Rev. Bras. Farm. 2005;17:79-15.
5. Maia FD, Iodes AF, Freitas RM. Atenção farmacêutica aos usuários do Centro de Atenção Psicossocial VI. Rev. Bras. Farm. 2006;55: 12-16.

6. Passos SRL, Camacho LAB. Características da clientela de um centro de tratamento para dependência de drogas. Rev. Saúde Pública 1998;32(1):64-71.

7. Pinho PH, Oliveira MAR, Almeida MM. A reabilitação psicossocial na atenção aos transtornos associados ao consumo de álcool e outras drogas: Uma estratégia possível? Rev. psiquiatr. clín. 2008;35(suppl. 1):82-8.

8. Spricigo JS, Alencastre MB. O enfermeiro de unidade básica de saúde e o usuário de drogas - um estudo em Biguaçú-SC. Rev. Latino-Am. Enfermagem. 2004;12(spe):427-32.

9. Morgado AF, Coutinho ESF. Dados de epidemiologia descritiva de transtornos mentais em grupos populacionais do Brasil. Cad. de Saúde Pública 1985;1(3):327-47.

10. Cipolle RJ, Strand LM, Morley PC, Frakes M. Resultados del ejercicio de la Atencion Farmacéutica. Pharm Care Esp 2000;2:94-106.

11. Onocko-Campos RT, Furtado JP. Entre a saúde coletiva e a saúde mental: um instrumental metodológico para avaliação da rede de Centros de Atenção Psicossocial (CAPS) do Sistema Único de Saúde. Cad. Saúde Pública 2006;22(5):1053-62.

12. Andreoli SB, Ronchetti SSB, Miranda ALP, Bezerra CRM, Magalhães CCPB, Martin D, et al. Utilização dos Centros de Atenção Psicossocial (CAPS) na cidade de Santos, São Paulo, Brasil. Cad. Saúde Pública 2004;20(3):836-44.

13. Alberola GEC, Cruz ME, Cruz T. Farmacovigilancia em aténción primária: experiencia en centro de salud. Rev OFIL 1991;2:85-8.

14. Lima SL, Soares BGO, Mari JJ. Saúde e doença mental em Pelotas, RS: dados de um estudo populacional. Rev. Psiquiatr. Clín. 1999;26(5):225-35.

15. Figlie N, Fontes A, Moraes E, Payá R. Filhos de dependentes químicos com fatores de risco biopsicossociais: necessitam de um olhar especial? Rev. Psiquiatr. Clín. 2004;31(2):53-62.

16. Lima MCP, Menezes PR, Carandina L, Cesar CLG, Barros MBA, Goldbaum M. Transtornos mentais comuns e uso de psicofármacos: impacto das condições socioeconômicas. Rev. Saúde Pública 2008;42(4):717-23. 17. Fernandes LA, Philomena MSF, Leite SMS. O perfil dos pacientes com transtornos mentais atendidos em unidade de saúde 24 horas de Curitiba. Boletim de Enfermagem 2007;1:14-29.

18. Secretaria de Saúde do Estado do Ceará. Manual de Procedimentos de Atenção Farmacêutica da Teoria a Prática. Fortaleza: Secretaria de Saúde do Estado do Ceará, 2003.

19. Lopes CS, Coutinho ESF. Transtornos mentais como fatores de risco para o desenvolvimento de abuso/ dependência de cocaína: estudo caso-controle. Rev. Saúde Pública. 1999;33(5):477-87.

20. Miranda SP, Vargas D. Satisfação de Pacientes de um Centro de Atenção Psicossocial Álcool e Drogas com o Atendimento do Enfermeiro. SMAD, Rev. Eletrônica 
Saúde Mental Álcool Drog. 2009;5(2):1-15.

21. Marcelo PA. Aplicação da versão em português do instrumento de avaliação de qualidade de vida da Organização Mundial da Saúde (WHOQOL-100). Rev. Saúde Pública. 1999;33(2):198-205.

22. Silva RF, Freitas RM. Investigação sobre o uso racional de medicamentos no município de Quixadá. Revista Eletrônica de Farmácia 2008;3:28-32.

23. Afonso GMT, Puerta FAMC. Auto valoración social, economica y profesional del farmacéutico comunitário. Rev OFIL 1991;5:298-303. 